Article

\title{
Vibrational Property of $\alpha$-Borophene Determined by Tip-Enhanced Raman Spectroscopy
}

\author{
Ping Zhang ${ }^{1,2,+}+\mathbb{D}$, Xirui Tian ${ }^{3,+}$, Shaoxiang Sheng ${ }^{1,2,+}$, Chen Ma ${ }^{1,2}$, Linjie Chen ${ }^{4}$, Baojie Feng ${ }^{1,2} \mathbb{D}$, \\ Peng Cheng ${ }^{1,2}$, Yiqi Zhang ${ }^{1,2}$, Lan Chen ${ }^{1,2}$, Jin Zhao ${ }^{5, * \mathbb{D}}$ and Kehui $\mathrm{Wu}^{1,2,6, * \mathbb{D}}$
}

1 Institute of Physics, Chinese Academy of Sciences, Beijing 100190, China; zhangping@iphy.ac.cn (P.Z.); sxsheng@iphy.ac.cn (S.S.); chma@iphy.ac.cn (C.M.); bjfeng@iphy.ac.cn (B.F.); pcheng@iphy.ac.cn (P.C.); yiqi.zhang@iphy.ac.cn (Y.Z.); lchen@iphy.ac.cn (L.C.)

2 School of Physical Sciences, University of Chinese Academy of Sciences, Beijing 100190, China

3 Department of Physics, University of Science and Technology of China, Hefei 230026, China; txr@mail.ustc.edu.cn

4 Department of Chemical Physics, School of Chemistry, University of Science and Technology of China, Hefei 230026, China; ljchen9@mail.ustc.edu.cn

5 CAS Key Laboratory of Strongly-Coupled Quantum Matter Physics, Department of Physics, University of Science and Technology of China, Hefei 230026, China

6 Songshan Lake Materials Laboratory, Dongguan 523808, China

* Correspondence: zhaojin@ustc.edu.cn (J.Z.); khwu@iphy.ac.cn (K.W.)

+ These authors contributed equally to this work.

\section{check for}

updates

Citation: Zhang, P.; Tian, X.; Sheng,

S.; Ma, C.; Chen, L.; Feng, B.; Cheng,

P.; Zhang, Y.; Chen, L.; Zhao, J.; et al. Vibrational Property of $\alpha$-Borophene Determined by Tip-Enhanced Raman Spectroscopy. Molecules 2022, 27, 834 . https://doi.org/10.3390/

molecules 27030834

Academic Editors: Takahiro Kondo, Iwao Matsuda and Josep

M. Oliva-Enrich

Received: 26 December 2021

Accepted: 11 January 2022

Published: 27 January 2022

Publisher's Note: MDPI stays neutral with regard to jurisdictional claims in published maps and institutional affiliations.

Copyright: (C) 2022 by the authors. Licensee MDPI, Basel, Switzerland. This article is an open access article distributed under the terms and conditions of the Creative Commons Attribution (CC BY) license (https:// creativecommons.org/licenses/by/ $4.0 /)$.

\begin{abstract}
We report a Raman characterization of the $\alpha$ borophene polymorph by scanning tunneling microscopy combined with tip-enhanced Raman spectroscopy. A series of Raman peaks were discovered, which can be well related with the phonon modes calculated based on an asymmetric buckled $\alpha$ structure. The unusual enhancement of high-frequency Raman peaks in TERS spectra of $\alpha$ borophene is found and associated with its unique buckling when landed on the $\mathrm{Ag}(111)$ surface. Our paper demonstrates the advantages of TERS, namely high spatial resolution and selective enhancement rule, in studying the local vibrational properties of materials in nanoscale.
\end{abstract}

Keywords: borophene; vibrational modes; tip-enhanced Raman spectroscopy; scanning tunneling microscopy; density functional theory calculations

\section{Introduction}

Borophene is an emerging two-dimensional (2D) material with novel properties, such as structural anisotropy [1], high thermal conductivity [2], metallicity [3,4], possible superconductivity [5] and polymorphism [4,6-8]. The unique polymorphism of borophene stems from the tremendous possible arrangements of hexagonal holes (HHs) in a planar triangular lattice. However, among the huge number of 2D borophene polymorphs that have been designed previously [7,9], only a few of them have been realized experimentally [10]. Using molecule beam epitaxial (MBE) to deposit boron on various metal substrates, different borophene polymorphs have been found on $\operatorname{Ag}(111)$ [4], $\operatorname{Ag}(100)$ [8], Cu(111) [11,12], $\operatorname{Ag}(110)$ [13], $\mathrm{Au}(111)$ [14], $\mathrm{Al}(111)$ [15] and $\operatorname{Ir}(111)$ [16,17]. Among them, the most studied phases are the $\beta_{12}$ and $\chi_{3}$ on $\mathrm{Ag}(111)$, and both of them can form large-area single phases with appropriate growth conditions [4]. Their structures and properties have been well established with different methods, such as in-situ Raman [18], angle-resolved photoemission spectroscopy (ARPES) [19,20] and high-resolution electron energy loss spectroscopy (HREELS) [21].

Among various 2D borophene polymorphs, the $\alpha$ phase with three-fold symmetry and $1 / 9 \mathrm{HH}$ density is particularly interesting, as it was predicted to be one of the most stable and fundamental borophene structures $[9,10]$. Previously, Zhong et al. reported the observation of small $\alpha$-phase domains coexisting with $\beta_{12}$ and $\chi_{3}$ phases in $\operatorname{Ag}(111)$ 
substrate [6]. Recently, Liu et al. reported the observation of bi-layer borophene on $\mathrm{Ag}(111)$, which was assigned to two covalently bonded $\alpha$-layers [22]. In both cases, the $\alpha$ phase only exists in small islands of typically nanometer size, and a complete $\alpha$-layer is still not available. This poses a great challenge to the understanding of the physical properties of $\alpha$ borophene, as even microscopic characterization techniques usually require samples of micrometer size. Thus, the properties of $\alpha$ borophene remain elusive so far.

In this paper, the vibrational properties of the $\alpha$ borophene were studied by combining scanning tunneling microscopy (STM) with tip-enhanced Raman spectroscopy (TERS). TERS allows one to detect the local vibrational properties with high spatial resolution $(<0.5 \mathrm{~nm})$ by the help of the strong localized electric field under the probe tip [23]. We obtained dramatically different Raman spectra from $\alpha$ borophene, as compared with those from other phases in the previous report [18]. DFT calculations reproduce the vibrational modes observed by the Raman spectra well, based on a buckled $\alpha$-phase model on $\operatorname{Ag}(111)$. Our results provide a fundamental data set for further studies of borophene and demonstrate the capability of TERS in the study of local properties of 2D materials

\section{Method}

All STM and TERS measurements were performed at $77 \mathrm{~K}$ using a home-made STMTERS system (located in Institute of Physics, CAS, Beijing, China), the base pressure being $10^{-8} \mathrm{~Pa}$. The single crystalline $\mathrm{Ag}(111)$ surface was cleaned by standard cycles of $\mathrm{Ar}^{+}$ion sputtering and annealing at $800 \mathrm{~K}$. Pure boron was evaporated from an e-beam evaporator to the $\mathrm{Ag}(111)$ substrate held at $570 \mathrm{~K}$ during deposition [4]. The TERS measurement was performed with side illumination and backscattering collection configuration [24]. A $532 \mathrm{~nm}$ laser was focused at the tunneling gap using aspheric lens attached to the side of the STM head in the ultrahigh vacuum chamber. The scattered Raman signals were dispersed by 1200 grooves/mm grating and collected by a liquid-nitrogen-cooled charge coupled device (CCD) (SP2300i, Princeton Instrument, Trenton, NJ, USA).

The first-principle calculations were performed within the framework of projectoraugmented wave (PAW) method [25], as implemented in Vienna Ab-initio Simulation Package (VASP) [26,27]. The electronic exchange-correlation interaction was described by Perdew-Burke-Ernzerhof (PBE) functional [28], and the van der Waals (vdW) correction was included using DFT-D3 method with Becke-Jonson damping [29]. We set a $500 \mathrm{eV}$ plane-wave cutoff and adopted a $12 \times 12 \times 1$ k-grid to sample the first Brillouin zone of the unit cell. All the atomic structures of borophene were fully relaxed on a two-layer $\operatorname{Ag}(111)$ surface until the changes in energy and force between each iteration step were respectively smaller than $10^{-8} \mathrm{eV}$ and $0.001 \mathrm{eV} / \AA$. To avoid the interlayer interaction, a $30 \AA$ vacuum interval was set up. With regard to phonon calculations, we employed the frozen-phonon method with $4 \times 4 \times 1$ supercell and $3 \times 3 \times 1 \mathrm{k}$-grid. The phonon dispersion was obtained based on the frozen-phonon results with the help of Phonopy [30]. Finally, all the models were shown using VESTA [31].

\section{Results and Discussion}

After the deposition of about $0.8 \mathrm{ML}$ boron atoms on the $\mathrm{Ag}(111)$ substrate, the $\mathrm{Ag}(111)$ surface was covered mainly with the $\beta_{12}$ borophene islands, which exhibited parallel Moiré stripes in parallel with the high-symmetry orientations of the $\mathrm{Ag}(111)$ substrate. Meanwhile, small domains with hexagonal Moiré patterns are frequently found to coexist with the $\beta_{12}$ phase, an example of which is shown in Figure 1a. The high resolution STM image in Figure 1b shows the hexagonal structure of this phase, with the lattice constant $a=0.52 \pm 0.01 \mathrm{~nm}$. This structure is consistent with the previously reported $\alpha$ borophene on $\operatorname{Ag}(111)$ [6]. 
(a)

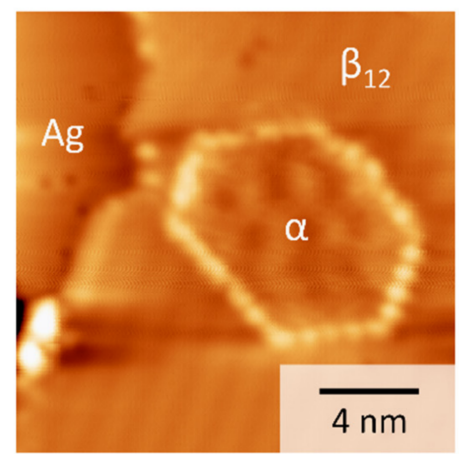

(c)

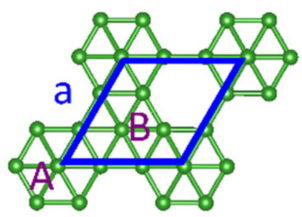

Without Sub. م-00:0000000 $\uparrow_{-0.16 \AA}^{+0.16 \AA}$

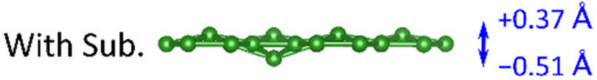

(b)



(d)

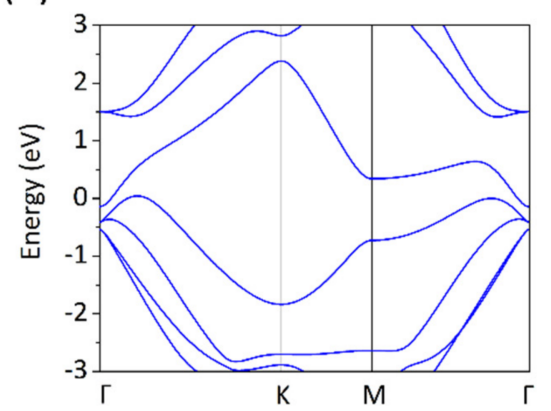

Figure 1. (a) STM image (1.3 V, $190 \mathrm{pA})$ of monolayer borophene sheet grown on $\mathrm{Ag}(111)$, showing an $\alpha$ domain between $\beta_{12}$ islands. (b) High-resolution STM image $(-0.8 \mathrm{~V}, 190 \mathrm{pA})$ of the surface of $\alpha$ borophene; the unit cell is marked by a blue rhombus. (c) The model of planar $\alpha$ phase, shown together with the symmetric buckled $\alpha$ phase (relaxed without $\operatorname{Ag}(111)$ substrate) and asymmetric buckled $\alpha$ phase (relaxed on $\operatorname{Ag}(111)$ substrate). (d) The simulated electronic band structure of asymmetric buckled $\alpha$ phase.

To understand the structure and properties of the $\alpha$ phase, it is worth noting that a completely flat $\alpha$ structure is unstable because of a large negative phonon frequency [9]. Instead, a symmetric and slightly buckled $\alpha$ phase with a vertical distance from the plane of about $\pm 0.16 \AA$ is found to be stable in the freestanding form [9]. The upward buckled boron atom in this model is marked by A, while the downward one is marked by B (Figure 1c). Furthermore, after relaxing the symmetric buckled $\alpha$ phase on the $\mathrm{Ag}(111)$ substrate, we found that the vertical distance of two buckled boron atoms from the plane further increased to $0.36 \AA$ and $-0.51 \AA$, exhibiting an asymmetric buckled structure. The electronic band structure of this asymmetric buckled phase is shown in Figure 1d. Its metallic properties are also consistent with previous STS result [6]. The asymmetric buckling is found to be critical for the vibrational properties of $\alpha$ borophene on $\operatorname{Ag}(111)$, as will be shown and discussed below.

TERS measurement was performed to obtain the vibrational information from the $\alpha$ borophene. As Figure 2a shows, when the STM tip is far from the surface, the far-field Raman signal is very weak due to the small Raman scattering cross-section of borophene [18]. When the STM probe tip is brought close to the surface of $\alpha$ borophene, a dramatic enhancement of Raman signal is observed, exhibiting a strong increment with the decrease in gap distance. The near-field TERS spectra clearly show a series of characteristic peaks, as illustrated in the background subtracted spectrum (the red curve in Figure $2 b$ ). Five strong peaks are found, located at $116.8 \mathrm{~cm}^{-1}, 157.3 \mathrm{~cm}^{-1}, 339.0 \mathrm{~cm}^{-1}, 702.6 \mathrm{~cm}^{-1}$ and $920.4 \mathrm{~cm}^{-1}$, together with three weak peaks at $406.4 \mathrm{~cm}^{-1}, 446.8 \mathrm{~cm}^{-1}$ and $1230.0 \mathrm{~cm}^{-1}$. For comparison, the TERS spectrum of $\alpha$ borophene is quite different from that of $\beta_{12}$ phase (blue curve in Figure 2b), as well as from that of $\chi_{3}$ phase reported in our previous study [18]. In particular, we observe significant enhancements of high-frequency peaks over $500 \mathrm{~cm}^{-1}$, in contrast with the cases of $\beta_{12}$ and $\chi_{3}$ phases, where only the low-frequency 
peaks are enhanced [18]. In view of the selective enhancement mechanism of TERS [18,23], only vibration modes that contain out-of-plane components can be enhanced effectively. For completely flat $2 \mathrm{D}$ borophene phases, such as $\beta_{12}$ and $\chi_{3}$, their high-frequency vibrational modes contain only in-plane components, and thus cannot be enhanced in TERS [18]. Therefore, we speculate that the obvious enhancement of the high-frequency Raman modes in $\alpha$ borophene implies that these vibrational modes contain out-of-plane components. This perfectly agrees with the fact that the $\alpha$ borophene is significantly buckled on $\operatorname{Ag}(111)$, according to DFT calculations.

(a)



(b)

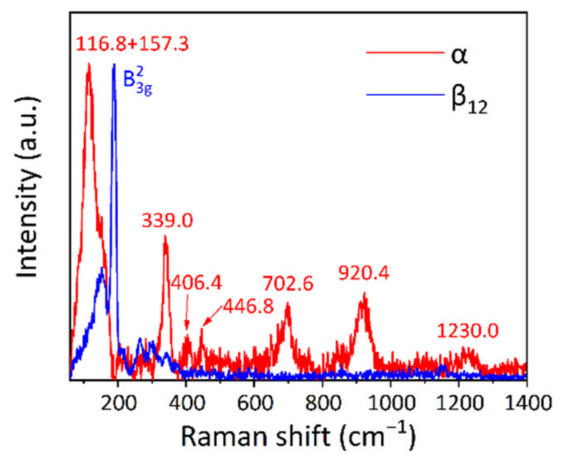

(c)
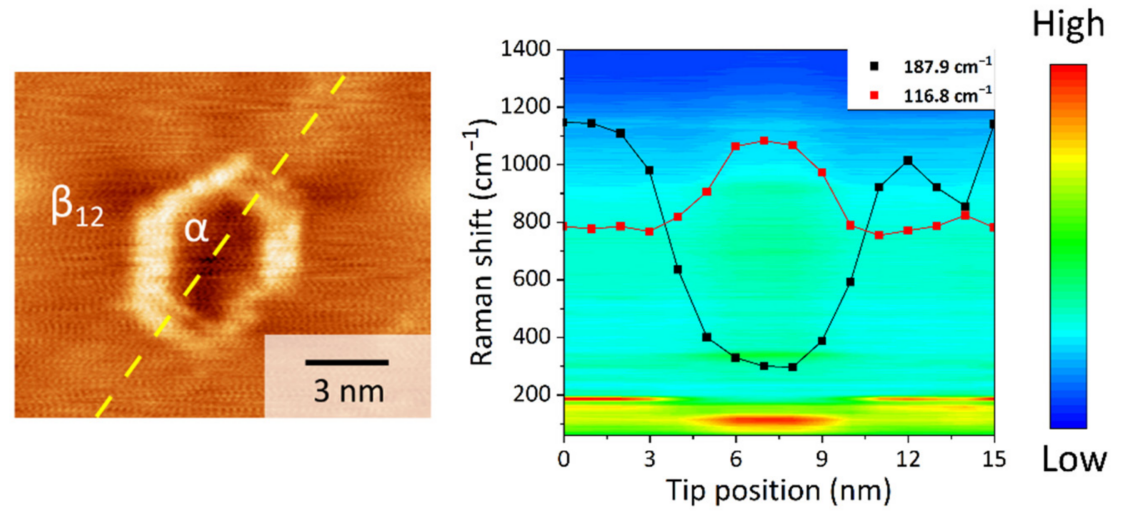

Figure 2. (a) Gap distance-dependent TERS spectra of $\alpha$ borophene $(10 \mathrm{~mW}, 0.3 \mathrm{~V}$, the accumulation time for each spectrum is $50 \mathrm{~s}$ ). The tip-sample distance was controlled by first decreasing the tunneling current from $300 \mathrm{pA}$ to $25 \mathrm{pA}$, and then the tip was retracted from the surface in $100 \mathrm{pm}$ steps with the feedback loop off. (b) Comparison of the TERS spectra of $\alpha$ and $\beta_{12}$ phases after background subtraction and normalization. (c) TERS spectra were taken along the yellow line, crossing the $\alpha$ borophene domain. The Raman intensity map was plotted in the right panel, where two dotted lines are the TERS intensity profiles of the two characteristic peaks of $\alpha$ borophene (red) and $\beta_{12}$ borophene (black), respectively.

We also emphasize that our TERS measurement renders Raman spectrum with extremely high spatial resolution. A series of TERS spectra were taken along the yellow line in the STM image shown in Figure $2 c$, crossing $\beta_{12}-\alpha-\beta_{12}$ regions as the $\alpha$ domain is surrounded by $\beta_{12}$ domains. One can see that when the STM tip moves from $\beta_{12}$ to $\alpha$ phase, the intensity of the characteristic $B_{3 g}{ }^{2}$ peak from the $\beta_{12}$ phase drop immediately, accompanied by the appearance of the characteristic $116.8 \mathrm{~cm}^{-1}$ peak from $\alpha$ borophene (the right panel of Figure 2c). Therefore, the high spatial resolution of TERS allows us to well separate the Raman signal of $\alpha$ borophene from that of surrounding $\beta_{12}$ phase, even though the size of the $\alpha$ borophene domain is only a few nanometers.

To account for these TERS peaks, we performed DFT calculations. The phonon spectra of both symmetric buckled $\alpha$ phase and asymmetric buckled $\alpha$ phase were simulated by VASP, respectively. The phonon spectrum of symmetric buckled $\alpha$ phase was found to largely deviate from our experimental TERS spectra. The phonon modes are completely 
absent in the vicinity of $116.8 \mathrm{~cm}^{-1}, 157.3 \mathrm{~cm}^{-1}, 339.0 \mathrm{~cm}^{-1}, 406.4 \mathrm{~cm}^{-1}, 1230.0 \mathrm{~cm}^{-1}$ at the $\Gamma$ point. In contrast, after relaxing the structure to the asymmetric buckled $\alpha$ phase, its symmetry changes from $D_{3 d}$ to $C_{3 v}$, causing the change of phonon spectrum. As shown in Figure $3 \mathrm{a}$, the phonon spectrum of asymmetric buckled $\alpha$ phase shows no obvious negative phonon frequencies, indicating a stable structure. Importantly, most peaks in experimental TERS spectra can be assigned to phonon modes at the $\Gamma$ point, as shown in Figure $3 \mathrm{~b}$. A detailed comparison of experimental and simulated peaks is shown in Table 1. The five low-frequency peaks located at $116.8 \mathrm{~cm}^{-1}, 157.3 \mathrm{~cm}^{-1}, 339.0 \mathrm{~cm}^{-1}, 406.4 \mathrm{~cm}^{-1}$, $446.8 \mathrm{~cm}^{-1}$ peak can be associated with $\mathrm{E}^{7}, \mathrm{E}^{6}, \mathrm{~A}_{1}^{4}, \mathrm{~A}_{1}^{3}, \mathrm{~A}_{2}^{3}$ and phonon modes, respectively, within a reasonable error range. The atomic displacements of these peaks, as shown in Figure $3 c$, are composed of nearly pure out-of-plane vibrational components, which accords with the selective enhancement rule in TERS. For the two high-frequency peaks located at $702.6 \mathrm{~cm}^{-1}$ and $920.4 \mathrm{~cm}^{-1}$, we can assign them to two phonon modes $\mathrm{E}^{4}$ and $\mathrm{E}^{3}$. The schematics of atomic displacements show that these two modes are composed of nearly in-plane vibrational components; however, the out-of-plane vibrational components still exist because of the two buckled boron atoms. Therefore, the enhancement of these two peaks in TERS accords with our model.

(a)



(b)

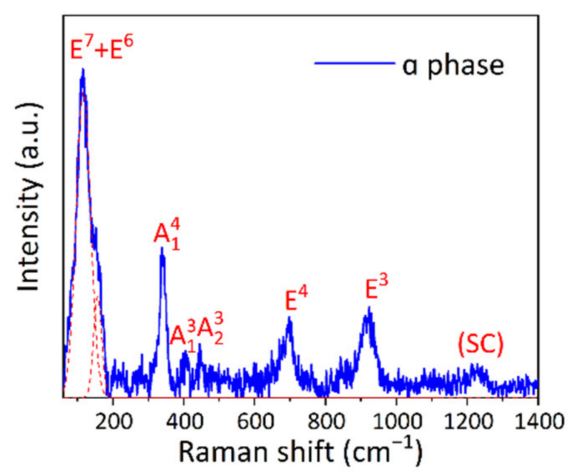

(c)

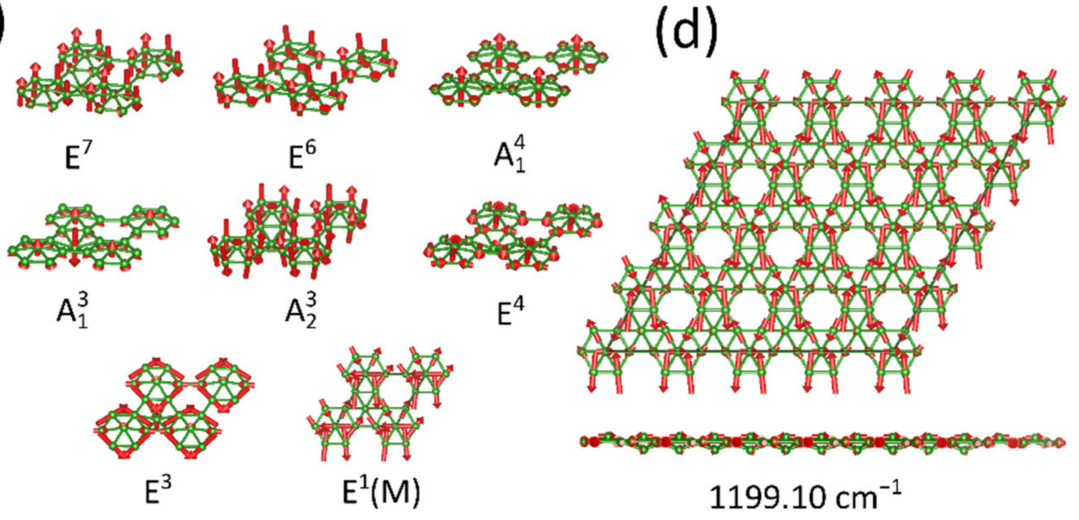

Figure 3. (a) The calculated phonon dispersion curves of asymmetric buckled $\alpha$ phase. (b) The near-field TERS signal of $\alpha$ phase, $\mathrm{E}^{7}$ and $\mathrm{E}^{6}$ peaks are fitted by red dashed curve. (c) Vibration modes of unit cell associated with TERS peaks. (d) The vibration mode of supercell associated with the $1230 \mathrm{~cm}^{-1}$ peak in TERS. 
Table 1. TERS modes of the $\alpha$ phase, as compared with the calculation, $\mathrm{cm}^{-1}$.

\begin{tabular}{ccc}
\hline TERS & Simulation & Modes \\
\hline 116.8 & 107.85 & $\mathrm{E}^{7}$ \\
157.3 & 188.6 & $\mathrm{E}^{6}$ \\
339.0 & 352.94 & $\mathrm{~A}_{1}^{4}$ \\
406.4 & 425.2 & $\mathrm{~A}_{1}^{3}$ \\
446.8 & 507.83 & $\mathrm{~A}_{2}^{3}$ \\
702.6 & 684.32 & $\mathrm{E}^{4}$ \\
920.4 & 907.16 & $\mathrm{E}^{3}$ \\
1230.0 & 1199.10 & Vibration of SC \\
\hline
\end{tabular}

Finally, the TERS peak located at $1230 \mathrm{~cm}^{-1}$ is about $90 \mathrm{~cm}^{-1}$ above the highest phonon mode at $\Gamma$ in the phonon spectrum. To account for this peak, we attribute it to a vibration mode of a supercell. Here, a $4 \times 4 \times 1$ supercell is considered because the periodicity of Moiré pattern of the $\alpha$ phase is about four times that of unit cell. Due to the Brillouin zone folding, the $\mathrm{M}$ point of the unit cell will be folded to the $\Gamma$ point in the new Brillouin zone of the $4 \times 4 \times 1$ supercell. The $\mathrm{E}^{1}$ mode at the $\mathrm{M}$ point contributes to a supercell vibrational mode with a frequency of $1199.10 \mathrm{~cm}^{-1}$ (Figure 3d), which matches our measurement better than any other modes contained in such a supercell. In addition to the frequency consistency, this mode also contains the out-of-plane vibration component. Therefore, it very likely corresponds to the $1230 \mathrm{~cm}^{-1}$ TERS peak.

\section{Conclusions}

In conclusion, we determine the characteristic Raman spectrum of $\alpha$ borophene with the help of high spatial resolution of TERS, combining with DFT calculations. All Raman peaks can be well associated with the phonon modes calculated based on an asymmetric buckled $\alpha$ structure. The unusual enhancement of high-frequency Raman peaks in TERS spectra of $\alpha$ borophene is also related to its unique buckling when landed on the $\operatorname{Ag}(111)$ surface. Our work provides not only the basic Raman characterization of the highly interesting $\alpha$ borophene, but also demonstrates the high prospect of TERS in studying local vibrational properties of nanoscale structures.

Author Contributions: P.Z., X.T. and S.S. have contributed in an equal manner in manuscript writing. Experiment performing, P.Z., S.S. and B.F.; DFT simulation, X.T. and L.C. (Linjie Chen); data analyzing, P.Z., C.M., P.C., Y.Z. and L.C. (Lan Chen); editing and interpretation, J.Z. and K.W. All authors have read and agreed to the published version of the manuscript.

Funding: This research was funded by the National Key R\&D Program of China (grant number 2021YFA1400500), National Natural Science Foundation of China (grands number 11825405, 12134019, 11974322, 12125408), and the Strategic Priority Research Program of the Chinese Academy of Sciences (grand number XDB30000000).

Informed Consent Statement: Not applicable.

Data Availability Statement: Data available via personal communication with proper reasons.

Conflicts of Interest: The authors declare no conflict of interest.

\section{References}

1. Feng, B.; Zhang, J.; Liu, R.-Y.; Iimori, T.; Lian, C.; Li, H.; Chen, L.; Wu, K.; Meng, S.; Komori, F.; et al. Direct evidence of metallic bands in a monolayer boron sheet. Phys. Rev. B 2016, 94, 041408. [CrossRef]

2. Xiao, H.; Cao, W.; Ouyang, T.; Guo, S.; He, C.; Zhong, J. Lattice thermal conductivity of borophene from first principle calculation. Sci. Rep. 2017, 7, 45986. [CrossRef] [PubMed]

3. Yang, X.; Ding, Y.; Ni, J. Ab initio prediction of stable boron sheets and boron nanotubes: Structure, stability, and electronic properties. Phys. Rev. B 2008, 77, 041402. [CrossRef]

4. Feng, B.; Zhang, J.; Zhong, Q.; Li, W.; Li, S.; Li, H.; Cheng, P.; Meng, S.; Chen, L.; Wu, K. Experimental realization of twodimensional boron sheets. Nat. Chem. 2016, 8, 563-568. [CrossRef] [PubMed] 
5. Penev, E.S.; Kutana, A.; Yakobson, B.I. Can two-dimensional boron superconduct? Nano Lett. 2016, 16, 2522-2526. [CrossRef]

6. Zhong, Q.; Zhang, J.; Cheng, P.; Feng, B.; Li, W.; Sheng, S.; Li, H.; Meng, S.; Chen, L.; Wu, K. Metastable phases of 2D boron sheets on Ag(111). J. Phys. Condens. Matter 2017, 29, 095002. [CrossRef]

7. Tang, H.; Ismail-Beigi, S. Novel precursors for boron nanotubes: The competition of two-center and three-center bonding in boron sheets. Phys. Rev. Lett. 2007, 99, 115501. [CrossRef]

8. Wang, Y.; Kong, L.; Chen, C.; Cheng, P.; Feng, B.; Wu, K.; Chen, L. Realization of regular-mixed quasi-1D borophene chains with long-range order. Adv. Mater. 2020, 32, e2005128. [CrossRef]

9. Wu, X.; Dai, J.; Zhao, Y.; Zhuo, Z.; Yang, J.; Zeng, X.C. Two-Dimensional boron monolayer sheets. ACS Nano 2012, 6, 7443-7453. [CrossRef]

10. Li, D.; Gao, J.; Cheng, P.; He, J.; Yin, Y.; Hu, Y.; Chen, L.; Cheng, Y.; Zhao, J. 2D Boron sheets: Structure, growth, and electronic and thermal transport properties. Adv. Funct. Mater. 2019, 30, 1904349. [CrossRef]

11. Chen, C.; Lv, H.; Zhang, P.; Zhuo, Z.; Wang, Y.; Ma, C.; Li, W.; Wang, X.; Feng, B.; Cheng, P.; et al. Synthesis of bilayer borophene. Nat. Chem. 2022, 14, 25-31. [CrossRef]

12. Wu, R.; Drozdov, I.K.; Eltinge, S.; Zahl, P.; Ismail-Beigi, S.; Bozovic, I.; Gozar, A. Large-Area single-crystal sheets of borophene on $\mathrm{Cu}(111)$ surfaces. Nat. Nanotechnol. 2019, 14, 44-49. [CrossRef] [PubMed]

13. Zhong, Q.; Kong, L.; Gou, J.; Li, W.; Sheng, S.; Yang, S.; Cheng, P.; Li, H.; Wu, K.; Chen, L. Synthesis of borophene nanoribbons on Ag(110) surface. Phys. Rev. Mater. 2017, 1, 021001. [CrossRef]

14. Kiraly, B.; Liu, X.; Wang, L.; Zhang, Z.; Mannix, A.J.; Fisher, B.L.; Yakobson, B.I.; Hersam, M.C.; Guisinger, N.P. Borophene synthesis on $\mathrm{Au}(111)$. ACS Nano 2019, 13, 3816-3822. [CrossRef]

15. Li, W.; Kong, L.; Chen, C.; Gou, J.; Sheng, S.; Zhang, W.; Li, H.; Chen, L.; Cheng, P.; Wu, K. Experimental realization of honeycomb borophene. Sci. Bull. 2018, 63, 282-286. [CrossRef]

16. Vinogradov, N.A.; Lyalin, A.; Taketsugu, T.; Vinogradov, A.S.; Preobrajenski, A. Single-Phase Borophene on $\operatorname{Ir}(111)$ : Formation, Structure, and Decoupling from the Support. ACS Nano 2019, 13, 14511-14518. [CrossRef]

17. Omambac, K.M.; Petrovic, M.; Bampoulis, P.; Brand, C.; Kriegel, M.A.; Dreher, P.; Janoschka, D.; Hagemann, U.; Hartmann, N.; Valerius, P.; et al. Segregation-Enhanced epitaxy of borophene on $\operatorname{Ir}(111)$ by thermal decomposition of borazine. ACS Nano 2021, 15, 7421-7429. [CrossRef]

18. Sheng, S.; Wu, J.B.; Cong, X.; Zhong, Q.; Li, W.; Hu, W.; Gou, J.; Cheng, P.; Tan, P.H.; Chen, L.; et al. Raman spectroscopy of two-dimensional borophene sheets. ACS Nano 2019, 13, 4133-4139. [CrossRef]

19. Feng, B.; Sugino, O.; Liu, R.Y.; Zhang, J.; Yukawa, R.; Kawamura, M.; Iimori, T.; Kim, H.; Hasegawa, Y.; Li, H.; et al. Dirac fermions in borophene. Phys. Rev. Lett. 2017, 118, 096401. [CrossRef]

20. Feng, B.; Zhang, J.; Ito, S.; Arita, M.; Cheng, C.; Chen, L.; Wu, K.; Komori, F.; Sugino, O.; Miyamoto, K.; et al. Discovery of 2D anisotropic dirac cones. Adv. Mater. 2018, 30, 1704025. [CrossRef]

21. Aizawa, T.; Suehara, S.; Otani, S. Phonon dispersion of a two-dimensional boron sheet on Ag(111). Phys. Rev. Mater. 2021, 5, 064004. [CrossRef]

22. Liu, X.; Li, Q.; Ruan, Q.; Rahn, M.S.; Yakobson, B.I.; Hersam, M.C. Borophene synthesis beyond the single-atomic-layer limit. Nat. Mater. 2022, 21, 35-40. [CrossRef]

23. Sheng, S.; Wu, J.B.; Cong, X.; Li, W.; Gou, J.; Zhong, Q.; Cheng, P.; Tan, P.H.; Chen, L.; Wu, K. Vibrational properties of a monolayer silicene sheet studied by tip-enhanced raman spectroscopy. Phys. Rev. Lett. 2017, 119, 196803. [CrossRef]

24. Sheng, S.; Li, W.; Gou, J.; Cheng, P.; Chen, L.; Wu, K. Low-Temperature, ultrahigh-vacuum tip-enhanced Raman spectroscopy combined with molecular beam epitaxy for in situ two-dimensional materials' studies. Rev. Sci. Instrum. 2018, $89,053107$. [CrossRef]

25. Blochl, P.E. Projector augmented-wave method. Phys. Rev. B 1994, 50, 17953-17979. [CrossRef] [PubMed]

26. Kresse, G.; Hafner, J. Ab initio molecular dynamics for open-shell transition metals. Phys. Rev. B 1993, 48, 13115-13118. [CrossRef] [PubMed]

27. Kresse, G.; Furthmüller, J. Efficiency of ab-initio total energy calculations for metals and semiconductors using a plane-wave basis set. Comput. Mater. Sci. 1996, 6, 15-50. [CrossRef]

28. Perdew, J.P.; Burke, K.; Ernzerhof, M. Generalized gradient approximation made simple. Phys. Rev. Lett. 1996, 77, 3865-3868. [CrossRef] [PubMed]

29. Grimme, S.; Antony, J.; Ehrlich, S.; Krieg, H. A consistent and accurate ab initio parametrization of density functional dispersion correction (DFT-D) for the 94 elements H-Pu. J. Chem. Phys. 2010, 132, 154104. [CrossRef]

30. Togo, A.; Tanaka, I. First principles phonon calculations in materials science. Scr. Mater. 2015, 108, 1-5. [CrossRef]

31. Momma, K.; Izumi, F. VESTA: A three-dimensional visualization system for electronic and structural analysis. J. Appl. Crystallogr. 2008, 41, 653-658. [CrossRef] 